\section{Post-acne hyperpigmentation: Evaluation of risk factors and the use of artificial neural net- work as a predictive classifier}

\author{
Firas Al-Qarqaz, ${ }^{1}$ Khaldon Bodoor, ${ }^{2}$ \\ Ala Baba, ${ }^{1}$ Ali Al-Yousef, ${ }^{3}$ Jihan \\ Muhaidat, ${ }^{1}$ Diala Alshiyab ${ }^{1}$ \\ ${ }^{1}$ Department of Dermatology, Jordan \\ University of Science and Technology, \\ Irbid; ${ }^{2}$ Department of Biotechnology \\ and Genetic Engineering, Faculty of \\ Science, Jordan University of Science \\ and Technology, Irbid; ${ }^{3}$ Computer \\ Department, Computer and Information \\ Technology College, Jerash University, \\ Jerash, Jordan
}

\begin{abstract}
Acne is common among young individuals. People with dark skin have a higher risk for developing pigmentary complications. Inflammation is an important factor in post-acne hyperpigmentation however other factors are also involved in developing this complication however these factors are not well studied. The aim of this study is to identify risk factors involved in post-acne hyperpigmentation. Clinical data related to acne, acne- related hyperpigmentation were collected. Data was analyzed for risk factors associated with acne pigmentation. Artificial neural network was used as predictive disease classifier for the outcome of pigmentation. Majority of patients in this study (339 patients) had dark skin phototypes (3 and 4). Post- acne hyperpigmentation was seen in more than $80 \%$ of patients. Females, darker skin color, severe acne, facial sites, and excessive sunlight exposure, squeezing or scratching lesions are important risk factors for post-acne hyperpigmentation. Post-acne hyperpigmentation is multifactorial. Several factors implicated in PAH are modifiable by adequate patient education (lesion trauma, excessive sunlight exposure). The use of ANN was helpful in predicting appearance of post-acne hyperpigmentation based on identified risk factors.
\end{abstract}

\section{Introduction}

Acne is one of most common conditions affecting skin. It affects almost all adolescents and up to $41 \%$ of adults may still suffer from acne disease. ${ }^{1,2}$ Pigmentary complications are important cosmetic concerns for all patients regardless of skin color and this can be more troublesome for the patient even more than the original skin condition. Patients with darker skin colors seem to be more likely to have pigmentary issues which should be an important consideration when evaluating these patients. ${ }^{3,4}$

The mechanisms by which acne can lead to hyperpigmentation are not well understood. Inflammation has an important role ${ }^{5}$ however other factors such as external trauma can also be involved. ${ }^{6}$ Post-acne hyperpigmentation can happen even in mild to moderate acne where inflammation is less severe. ${ }^{7}$

Paracrine signaling between keratinocytes, fibroblasts, and melanocytes plays an important role in skin hyperpigmentation disorders. ${ }^{8}$ Keratinocytes secrete multiple cytokines which act on melanocytes. ET-1 paracrine signaling pathways have been associated with the pathogenesis of seborrheic keratosis and senile lentigos, ${ }^{9,10}$ whereas deregulation of ET1signaling pathways is linked to hypopigmentation disorders such as vitiligo. ${ }^{11}$

In the current study, we examined the effect of patient factors (sex, skin color, and lesion trauma) as well as other factors including disease-related (severity, duration, site), treatment and the effect of sun light exposure on post-acne hyperpigmentation. Further, risk factors which show a significant impact on post-acne hyperpigmentation were used as inputs to build an ANN predictive classifier of the disease.

\section{Materials and methods}

A total of 339 patients were included in this study. Patients were recruited from dermatology out-patient clinics. Patients were examined for their acne including duration of acne, distribution of lesions, severity (The classification of acne severity was based on the Investigator Global Acne Severity Scale as following: 0 clear, 1 almost clear, 2 mild, 3 moderate, 4 severe) and presence of hyperpigmentation. Skin type was recorded for all patients. Demographic data including age and sex were also recorded.

Data analysis was done using SPSS software version 21 . P value $\leq 0.05$ is considered significant.

Collected data was then analyzed utilizing Artificial Neural Network (ANN). This is a computational method that mimic the human brain neural networks to predict the class of unseen samples. The networks contain nodes (neurons) that are organized in layers, each node in a layer $\mathrm{K}$ relates to all nodes in the layer K-1 (edge). The edge
Correspondence: Firas Al-Qarqaz, Department of dermatology, Jordan University of Science and Technology, PO Box 3030, Irbid, Jordan.

Tel.: +962790562121.

E-mail: fqarqaz@just.edu.jo.

Key words: Acne, Post-acne hyperpigmentation, Artificial neural network, Classification.

Contributions: FAQ: conception and design of the study, patient inclusion, monitoring study conduct, writing and drafting the paper; $\mathrm{KB}$ : conception and design of the study, writing and drafting the paper; $\mathrm{AB}$ : patient inclusion, data collection, drafting the work or revising it critically for important intellectual content; AAY: data analysis, figure production; ANN: design and production, writing and drafting the paper; JM, patient inclusion, data collection, drafting the work or revising it critically for important intellectual content; DA, patient inclusion, data collection, drafting the work or revising it critically for important intellectual content. All authors: final approval of version to be published and agreement to be accountable for all aspects of the work.

Conflict of interest: The authors declare no potential conflict of interest.

Funding and ethical approval: The study was supported and approved by the deanship of research at Jordan University of Science and Technology number 2018-0207. The study was approved by the ethical committee (IRB number: 164-2018).

Consent for publication: Not required.

Availability of data and material: Data and materials are available by the authors.

Please cite this article as: Al-Qarqaz F, Bodoor K, Baba A, et al. Post-acne hyperpigmentation: Evaluation of risk factors and the use of artificial neural network as a predictive classifier. Dermatol Rep 2021;13:8223.

Received for publication: 25 June 2019. Accepted for publication: 25 May 2020.

This work is licensed under a Creative Commons Attribution-NonCommercial 4.0 International License (CC BY-NC 4.0).

C Copyright: the Author(s), 2021

Licensee PAGEPress, Italy

Dermatology Reports 2021; 13:8223

doi:10.4081/dr.2021.8223

between two neurons carries a weight (number) which represents the knowledge of the network. Building ANN is done through three main steps: First, the data is divided into three subsets (training, validation and testing). Subsequently, training 
step (repeated multiple times), is used as an input to the network, to adapt the weights of the edges which encode the knowledge in the ANN. This is done using the proper learning method like Scaled Conjugate Gradient Back Propagation algorithm (SCGBP) as the learning method and the tan-sigmoid function as the activation function. The generated trained-network is, subsequently, validated using validation subset to assess the performance of the network. Finally, the validated neural network is tested using testing subset. The built ANN is a classifier which can be utilized to predict the influence of multiple risk factors on the development of post-acne hyperpigmentation. In this study, risk factors which show significant $\mathrm{P}$ value were used as the input factors to create a predictive ANN. The data was divided randomly into three groups (training $70 \%$, validation $15 \%$, and testing $15 \%)$. The performance of the neural network is given in the form of confusion matrix showing sensitivity, specificity, accuracy, false positive and false negative results. These terms are defined as following. Sensitivity: number of samples correctly classified/total number of pigmented samples. Specificity: number of non-pigmented samples correctly classified/total number of non-pigmented samples. Accuracy: number of samples correctly classified/total number of samples. False positive: number of non-pigmented sample falsely classified/total number of pigmented samples. False Negative: number of pigmented sample falsely classified/total number of non-pigmented sample. The confusion matrix was evaluated for accuracy using the Receiver Operating Characteristics (ROC) plot. The study was approved by the ethical committee (IRB number: 164-2018).

\section{Results}

A total of 339 patients were recruited for the study. There were 90 patients with mild acne and 249 patients with moderate to severe acne. 242 cases $(71.3 \%)$ had acne related pigmentation (42 patients with mild acne, 200 patients with moderate to severe acne) while 97 cases (28.7\%) did not have post acne pigmentation. Of cases with no pigmentation, $48(49.5 \%)$ patients had mild acne while the remainder 49 patients $(51.5 \%)$ had moderate to severe acne. The predominant skin types are Fitzpatrick types 3 and 4 accounting for around $77.5 \%$ of all patients. The main clinical features are shown in Table 1.

Facial sites were predominantly affected by pigmentation $(83 \%)$ compared to trunk $(17 \%)$. The cheeks and forehead were the most-commonly affected site while the nose was the least affected site (Figure 1).

Several clinical variables could be linked to post acne pigmentation. In this study we examined 13 different parameters to assess their effect on pigmentation with acne lesions. Dark Skin type (3 and 4) was shown to be more associated with pigmentation when compared to type $2(\mathrm{P}=0.006)$. Females were more likely to have post acne pigmentation compared to males $(\mathrm{P}=0.008)$ although in this study females outnumbered males by almost 4 folds. Additionally, we observed a strong correlation between sunlight exposure and development of post acne pigmentation $(\mathrm{P}=0.005)$. Facial sites (cheeks and forehead) were found to be more affected by pigmentation $(\mathrm{P}=0.0001)$, a further testament to the correlation between sunlight exposure and pigmentation observed in this study. Acne severity (moderate to severe) was more associated with pigmentation when compared to mild acne cases $(\mathrm{P}=0.0001)$. An important finding of this study was the observation of a strong correlation between having itchy lesions and the practice of squeezing/scratching lesions and pigmentation. In support of these findings, we also observed that patients who more frequently scratch their acne lesions tend to have more pigmentation $(\mathrm{P}=0.0001)$.

The other clinical parameters analyzed including age, duration of acne, use of sun block, type of treatment used, were not found to have significant correlation with post-acne hyperpigmentation in this cohort of patients.

In this study we used the clinical parameters that show significant correlation with post acne pigmentation (Sex, skin type, sunlight exposure, site, severity, itch, squeeze, scratch and frequency of scratching) as an input to the neural network. The output of the network is defined as 0 (absence of pigmentation) and 1 (presence of pigmentation). The ANN for this study is shown in Figure 2.

The predictive power of the designed

\section{Sites of Pigmentation}

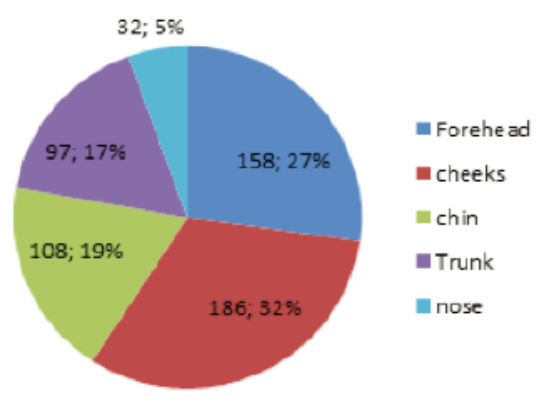

Figure 1. Site distribution of post-acne pigmentation.

Table 1. Main clinical features of patients.

\begin{tabular}{|c|c|c|c|}
\hline & $\begin{array}{l}\text { Mild acne } \\
(\mathrm{N}=90)\end{array}$ & $\begin{array}{l}\text { Moderate to severe acne } \\
\qquad(\mathrm{N}=249)\end{array}$ & $\begin{array}{l}\text { All groups } \\
(\mathrm{N}=339)\end{array}$ \\
\hline Average age & 22.5 years & 21.9 years & 22 years \\
\hline $\begin{array}{c}\text { Sex number } \\
\text { Female } \\
\text { Male }\end{array}$ & $\begin{array}{l}67 \\
23\end{array}$ & $\begin{array}{c}207 \\
42\end{array}$ & $\begin{array}{c}274(80.8 \%) \\
65(19.2 \%)\end{array}$ \\
\hline $\begin{array}{c}\text { Skin type, N (\%) } \\
2 \\
3 \\
4 \\
5\end{array}$ & $\begin{array}{l}10(11.1) \\
45(50) \\
31(34.5) \\
4(4.4)\end{array}$ & $\begin{array}{c}62(24.9) \\
147(59) \\
40(16.1) \\
0(0)\end{array}$ & $\begin{array}{l}72(21.3) \\
192(56.7) \\
71(20.9) \\
4(0.01)\end{array}$ \\
\hline $\begin{array}{l}\text { Post acne pigmentation, } \mathrm{N}(\%) \\
\text { Yes } \\
\text { No }\end{array}$ & $\begin{array}{l}42(46.7) \\
48(53.3)\end{array}$ & $\begin{array}{c}200(80.3) \\
49(19.7)\end{array}$ & $\begin{array}{c}242(71.3) \\
97(28.7)\end{array}$ \\
\hline Average duration of acne, months & 44.6 & 47.5 & 46.7 \\
\hline
\end{tabular}


ANN was validated using confusion matrix. The results from training, test, and validation of the matrix are shown in Figure 3. The classifier confusion matrix was able to show $83.8 \%$ accuracy in predicting appearance of pigmentation with 224 cases correctly classified with $92.6 \%$ sensitivity and $14.2 \%$ false positive rate. On the other hand, for cases which had no post acne pigmentation, 60 cases were correctly matched using the ANN with $61.9 \%$ specificity and $23.1 \%$ false negative rate. The overall results are shown in the all-confusion matrix shown in Figure 3.

The confusion matrix was evaluated for accuracy using ROC plots. Figure 4 shows ROC plots for training, test, validation and the overall results matrix. All matrices were shown to have good performance levels indicating that the matrix was sensitive and specific in predicting both the appearance and absence of pigmentation as shown in Figure 4.

\section{Discussion}

Pathogenesis of acne is multifactorial. Sebum secretion from sebaceous glands plays a central role in setting stage for acne development and this explains the predominance of acne lesions in areas rich in sebaceous glands. ${ }^{12}$ Inflammation is another key factor and this seems to be an early event that is even present in lesions without clinically obvious inflammation. ${ }^{13}$ Propionibacterium acnes contributes to inflammation within the pilosebaceous unit (PSU) through inflammasome activation and eventually IL-1 beta and TH-17 activation. ${ }^{14}$

The complications associated with acne include scarring and pigmentary complications which affects almost all patients. ${ }^{15}$ Acne scars tend to be of the depressed type and are generally associated with more severe grades of acne. The treatment of scars can be achieved by various procedures including micro needling, lasers, peels, fillers and others. ${ }^{16,17}$

Pigmentary complications of acne are an important complication that needs to be addressed. In patients with dark skin colors this problem can be more devastating than their original acne. ${ }^{18}$ Several factors are known to be associated with development of post-acne hyperpigmentation. Inflammation seems to be an important factor and the pigmentation following acne is usually referred to as post-inflammatory hyperpigmentation (PIH) which implies that pigmentation is caused by the preceding inflammatory response. However, this seems to be an oversimplification as several other factors may also be contributing including skin color, effect of treatment among other possible factors that were examined in this study. Hence, we prefer the use of post-acne hyperpigmentation (PAH) as this is includes other possible factors other than inflammation contributing to pigmentation appearing after acne lesions.
In this cohort, there was a predominance of females seeking treatment for their acne compared to males ( $80.8 \%$ vs $19.2 \%)$. Other previous studies have shown similar female predominance among acne patients visiting clinics. ${ }^{7}, 19$ Females are generally more aware and affected by their appearance and may be more motivated to seek medical treatment ${ }^{20}$ In this study, Females



Figure 2. Design of an artificial neural network (ANN) for the prediction of post-acne pigmentation. The ANN includes risk factors as the input layer, a hidden layer and the output layer represented by 0 (absence of pigmentation) or 1 (presence of pigmentation).
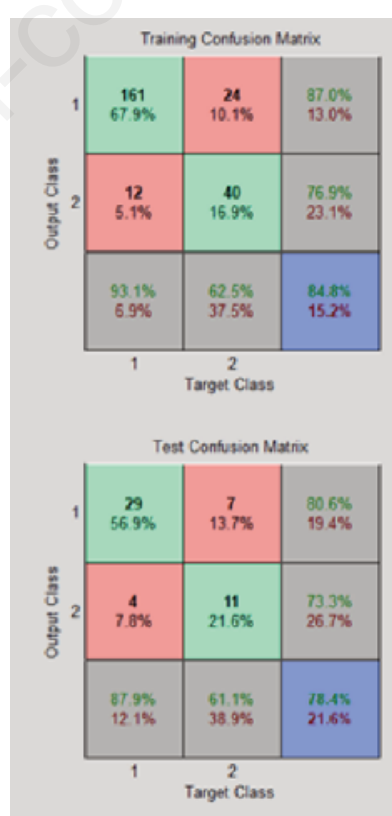


Figure 3. The confusion matrix for the selected features. In the matrix, target class represents observed Pigmented cases and output class represents predicted pigmented cases. 
with darker skin color were more likely to develop PAH. The appearance of pigmentation following acne lesions in dark skin has been shown by several other studies. 7,19,21 On the other hand, the study by RuizMaldonado and Orozco-Covarrubias indicates an individual chromatic tendency regardless of skin phototype which further complicates the subject of skin color and pigmentation. ${ }^{22}$

Most patients included in this study had moderate to severe acne. The reason for this is that our clinics are located within a referral hospital which means that patients with more severe acne are more likely to be seen in our clinics while patients with milder forms of acne are more likely to be managed by primary care physicians. This also could explain the relatively longer duration of acne disease seen in this cohort where patients are more likely to have tried treatment of their acne by primary care physicians prior to treatment in our clinics.

An important finding of this study is that lesion physical trauma (squeezing, scratching) was strongly related to appearance of PAH. Several studies suggest a link between physical trauma and the pathogenesis of several skin hyperpigmentation disorders. These include friction melanosis, nipple-areolar complex pigmentation, pigmentation following dermabrassion and lasers among others. ${ }^{23}$ Trauma-induced paracrine cytokine stimulation as a causative factor in skin hyperpigmentation was investigated by Kurita et al. ${ }^{24}$ In their work, the effect of cyclic stretch on the expression of keratinocyte- and fibroblastderived melanogenic paracrine cytokines was studied in a primary human skin model. Their results show that cyclic stretch of keratinocytes caused a marked upregulation of the key paracrine cytokine ET-1. Further, the study found that ET-1 upregulation is correlated with the length and frequency of the stretch. This finding is consistent with our results showing a strong association between physical trauma of acne lesions in the form of squeezing and scratching and the frequency of repeated trauma with the appearance of PAH. A role for physical trauma(referred to excoriation) in the development of post-acne hyperpigmentation is further supported by a study of acne patients from seven Asian countries. ${ }^{9}$ The effect of physical trauma in the development of postacne hyperpigmentation is also validated by a TCA-induced PIH model. ${ }^{25}$ In this model, a cohort of mainly dark skin type individuals were subjected to epidermal injury using a $35 \%$ trichloroacetic acid (TCA) solution and the results clearly show that injuryinduced pigmentation lesions are inditnguishable from acne-induced pigmented lesions. a recent study by Passeron et al. showed that epidermal injury in the form of suction blisters applied to darker skin induced hyperpigmentation. ${ }^{26}$ Additionally, their results show that hyperpigmentation could be prevented provided the suction blister areas are protected from sun exposure. Having itch due to acne is important in driving patients to scratch or squeeze lesions. This finding is of extreme importance because the practice of lesion trauma by squeezing and/or excoriation can be minimized with adequate patient advice and pointing out the increased risks of $\mathrm{PAH}$ especially in higher risk patients Additionally, the use of treatment measures that can reduce itch may be an important missing part of the treatment for patients with higher risk for having PAH The mechanisms of physical trauma -induced hyperpigmentation is complex and future studies should be geared towards the identification of the signaling pathways involved in ET-1 regulation of melanocytes in addition to other paracrine signaling molecules.

In this study, acne severity was shown to have a stronger association with occurrence of PAH. This can be explained by the presence of more intense inflammation in cases with moderate to severe acne. We found no previous studies that compared PAH according to acne severity grade. The sites of PAH were similar to sites reported by Abad-Casintahan et.al with cheeks and forehead skin were affected more than other sites. $^{7}$ This could be explained by that fact that these sites are more exposed to sun-

light, an important factor that stimulates melanocytes. Additionally, facial acne is more amenable to trauma by patient scratching and squeezing, a common practice amongst patients in this cohort. Interestingly, in this study we found no correlation between duration of acne lesions and PAH. This could be explained by the fact that the average duration of acne for patients was around 3 years and half which is relatively a long time.

Although treatment of acne is expected to influence risk of $\mathrm{PAH}$, we found no difference in appearance of $\mathrm{PAH}$ and treatment used by patients in this study. Furthermore, patients not using any treatment for their acne had similar risk of developing PAH. Again, this could be due to the fact that PAH is associated with several other factors that contribute to the ultimate risk of developing PAH.

The effects of sunlight exposure on pigmentation of exposed skin are well established. ${ }^{27,28}$ In the current study facial skin was more affected by PAH compared to non-exposed truncal skin. There was no protective effect from use of sun block, however this could be explained by the inadequate use of sun block by most patients. Only around 1/3 (149 patients) of patients in this cohort indicated that they were using sun block always or most of the time. This is an important point to take into consideration when managing acne patients with risk of developing PAH. These patients should be given adequate counselling regarding the importance of adequate and

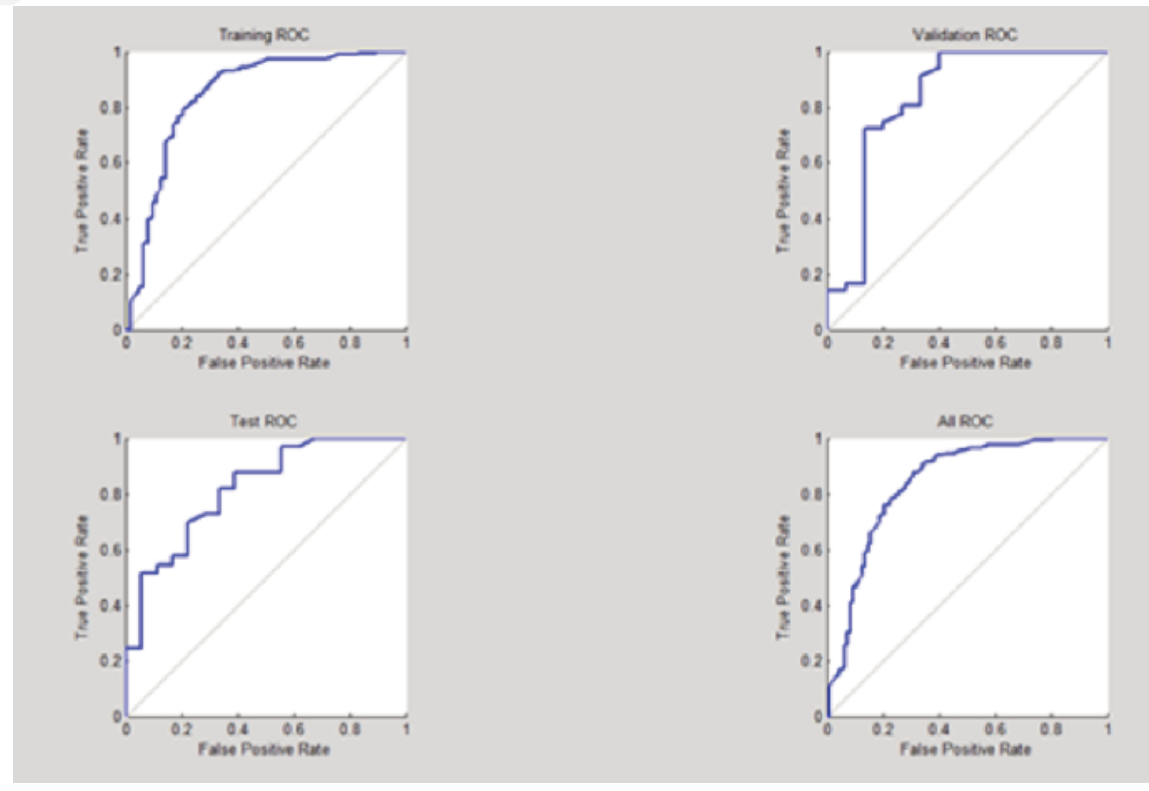

Figure 4. The Receiver Operating Characteristic (ROC) curve for confusion matrices.

[Dermatology Reports 2021; 13:8223] 
proper use of sun protecting creams.

Given the complexity of PAH and the many factors that may influence pigmentation we used ANN to build a predictive classifier. ANN was used in the predictive diagnosis of several skin disorders. ${ }^{29,30}$ Based on the risk factors identified from this study, the ANN was able to give an accurate risk prediction of $\mathrm{PAH}$.

\section{Conclusions}

Post-acne hyperpigmentation is a frequent and important complication of acne. Several risk factors are involved in the development of PAH including inflammation, dark skin color especially in females with severe facial acne, sunlight exposure and lesion trauma. Adequate patient education on these risk factors can help in reducing the complications of acne. The use of $\mathrm{ANN}$ is helpful in predicting PAH and can be a useful diagnostic tool in dermatology practice.

\section{References}

1. Wolkenstein P, Machovcová A, Szepietowski JC, et al. Acne prevalence and associations with lifestyle: a crosssectional online survey of adolescents/young adults in 7 european countries. J Eur Acad Dermatol Venereol. 2018;32:298-306.

2. Perkins AC, Maglione J, Hillebrand $\mathrm{GG}$, et al. Acne vulgaris in women: prevalence across the life span. J Womens Health (Larchmt). 2012;21:223-30.

3. Chua-Ty G, Goh CL, Koh SL. Pattern of skin diseases at the national skin centre (Singapore) from 1989-1990. Int J Dermatol 1992;31:555-9.

4. Abanmi A, Al-Enezi M, Al Hammadi A et al.S urvey of acne-related postinflammatory hyperpigmentation in the middle east. J Dermatolog Treat 2018;14:1-4.

5. Tomita Y, Maeda K, Tagami H. Leukotrienes and thromboxane B2 stimulate normal human melanocytes in vitro: possible inducers of postinflammatory pigmentation. Tohoku J Exp Med 1988;156:303-4.

6. Lamel SA, Rahvar M, Maibach HI. Postinflammatory hyperpigmentation secondary to external insult: an overview of the quantitative analysis of pigmentation. Cutan Ocul Toxicol
2013;32:67-71.

7. Abad-Casintahan F, Chow SK, Goh CL et al. Frequency and characteristics of acne-related post-inflammatory hyperpigmentation. J Dermatol 2016;43:8268.

8. Imokawa. G. Autocrine and paracrine regulation of melanocytes in human skin and in pigmentary disorders. Pigment Cell Res 2004;17:96-110.

9. Teraki E, Tajima S, Manaka I et al. Role of endothelin-1 in hyperpigmentation in seborrhoeic keratosis. Br J Dermatol 1996;135:918-23.

10. Murase D, Hachiya A, Kikuchi-Onoe $\mathrm{M}$, et al. Cooperation of endothelin-1 signaling with melanosomes plays a role in developing and/or maintaining human skin hyperpigmentation. Biol Open 2015;4:1213-21.

11. Kitamura R, Tsukamoto K, Harada K, et al. Mechanisms underlying the dysfunction of melanocytes in vitiligo epidermis: role of $\mathrm{SCF} / \mathrm{KIT}$ protein interactions and the downstream effector, MITF-M. J Pathol 2004;202:463-75.

12. Zouboulis CC, Jourdan E, Picardo M. Acne is aninflammatory disease and alterations of sebum Composition initiate acne lesions. J Eur Acad Dermatol Venereol 2014;28:527-32.

13. Dreno B, Gollnick HP, Kang $\mathrm{S}$ et al. Global alliance to improve outcomes in acne. Understanding innate immunity and inflammation in acne: implications for management. J Eur Acad Dermatol Venereol 2015;29:3-11.

14. Thiboutot DM. Inflammasome activation by propionibacterium acnes: the story of IL-1 in acne continues to unfold. J Invest Dermatol 2014; 134:595-9.

15. Layton AM, Henderson CA, Cunliffe WJ. A clinical evaluation of acne scarring and its incidence. Clin Exp Dermatol 1994;19:303-8.

16. Goodman GJ. Treatment of acne scarring. Int J Dermatol 2011;50:1179-94.

17. Al Qarqaz F, Al-Yousef A. Skin microneedling for acne scars associated with pigmentation in patients with dark skin. J Cosmet Dermatol 2018;17:39095.

18. Darji K, Varade R, West D, et al. Psychosocial impact ofpostinflammatory hyperpigmentation in patients with acne vulgaris. J Clin Aesthet Dermatol 2017;10:18-23.

19. Davis EC, Callender VD. Postinflammatory hyperpigmentation: a review of the epidemiology, clinical features, and treatment options in skin of color. J Clin Aesthet Dermatol 2010;3:20-31.

20. Zeichner JA, Baldwin HE, CookBolden FE, et al. Emerging issues in adult female acne. J Clin Aesthet Dermatol 2017;10:37-46.

21. Silpa-Archa N, Kohli I, Chaowattanapanit $\mathrm{S}$, et al. Postinflammatory hyperpigmentation: A comprehensive overview: epidemiology, pathogenesis, clinical presentation, and noninvasive assessment technique. J Am Acad Dermatol 2017;77:591-605.

22. Ruiz-Maldonado R, OrozcoCovarrubias ML. Postinflammatory hypopigmentation and hyperpigmentation. Semin Cutan Med Surg 1997;16: 36-43.

23. Yoshimura K, Momosawa A, Watanabe A, et al. Cosmetic color improvement of the nipple-areola complex by optimal use of tretinoin and hydroquinone. Dermatol Surg 2002;28:1153-7.

24. Kurita M, Okazaki M, Fujino T et al. Cyclic stretch induces upregulation of endothelin-1 with keratinocytes in vitro: possible role in mechanical stressinduced hyperpigmentation. Biochem Biophys Res Commun 2011;409:103-7.

25. Isedeh P, Kohli I, Al-Jamal M, et al. An in vivo model for postinflammatory hyperpigmentation: an analysis of histological, spectroscopic, colorimetric and clinical traits. Br J Dermatol 2016;174: 862-8.

26. Passeron T, Nouveau S, Duval C, et al. Development and validation of a reproducible model for studying post-inflammatory hyperpigmentation. Pigment Cell Melanoma Res 2018;31:649-52.

27. Tomita Y, Maeda K, Tagami H. Melanocyte-stimulating properties of arachidonic acid metabolites: possible role in postinflammatory pigmentation. Pigment Cell Res 1992;5:357-61.

28. Serre C, Busuttil V, Botto JM. Intrinsic and extrinsic regulation of human skin melanogenesis and pigmentation. Int $\mathrm{J}$ Cosmet Sci 2018;40:328-47.

29. Cheng B, Stanley RJ, Stoecker WV, et al. Analysis of clinical and dermoscopic features for basal cell carcinoma neural network classification. Skin Res Technol 2013;19:e217-22.

30. Hirota M, Kouzuki H, Ashikaga T, et al. Artificial neural network analysis of data from multiple in vitro assays for prediction of skin sensitization potency of chemicals. Toxicol in Vitro 2013;27:1233-46. 